Open Access

\title{
Acupuncture for chronic fatigue syndrome and idiopathic chronic fatigue: a multicenter, nonblinded, randomized controlled trial
}

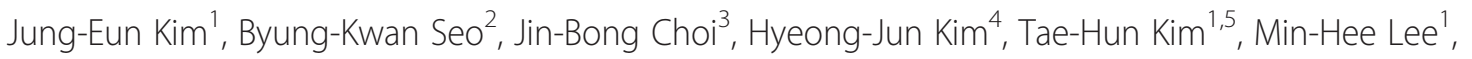
Kyung-Won Kang ${ }^{1}$, Joo-Hee Kim', Kyung-Min Shin'', Seunghoon Lee ${ }^{1,6}$, So-Young Jung ${ }^{1}$, Ae-Ran Kim',

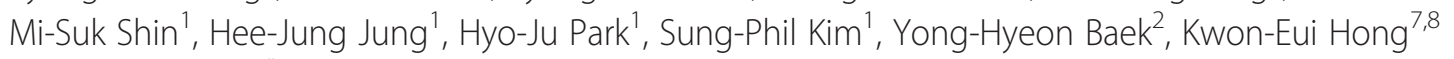
and Sun-Mi Choi ${ }^{*}$

\begin{abstract}
Background: The causes of chronic fatigue syndrome (CFS) and idiopathic chronic fatigue (ICF) are not clearly known, and there are no definitive treatments for them. Therefore, patients with CFS and ICF are interested in Oriental medicine or complementary and alternative medicine. For this reason, the effectiveness of complementary and alternative treatments should be verified. We investigated the effectiveness of two forms of acupuncture added to usual care for CFS and ICF compared to usual care alone.

Methods: A three-arm parallel, non-blinded, randomized controlled trial was performed in four hospitals. We divided 150 participants into treatment and control groups at the same ratio. The treatment groups (Group A, body acupuncture; Group B, Sa-am acupuncture) received 10 sessions for 4 weeks. The control group (Group C) continued usual care alone. The primary outcome was the Fatigue Severity Scale (FSS) at 5 weeks after randomization. Secondary outcomes were the FSS at 13 weeks and a short form of the Stress Response Inventory (SRI), the Beck Depression Inventory (BDI), the Numeric Rating Scale (NRS), and the EuroQol-5 Dimension (EQ-5D) at 5 and 13 weeks.

Results: Group A showed significantly lower FSS scores than Group $C$ at 5 weeks $(P=0.023)$. SRI scores were significantly lower in the treatment groups than in the control group at 5 (Group $A, P=0.032 ; B, P<0.001$ ) and 13 weeks (Group A, $P=0.037 ; B, P<0.001$ ). Group B showed significantly lower BDI scores than Group $C$ at 13 weeks $(P=0.007)$. NRS scores from the treatment groups were significantly reduced compared to control at 5 (Group A and B, $P<0.001$ ) and 13 weeks (Group A, $P=0.011 ; B, P=0.002$ ).
\end{abstract}

Conclusions: Body acupuncture for 4 weeks in addition to usual care may help improve fatigue in CFS and ICF patients.

Trial registration: Clinical Research Information Service (CRIS) KCT0000508; Registered on 12 August 2012.

Keywords: acupuncture, fatigue, randomized controlled trials as topic

\footnotetext{
* Correspondence: smchoi@kiom.re.kr

${ }^{1}$ Acupuncture, Moxibustion \& Meridian Research Group, Korea Institute of Oriental Medicine, Daejeon, South Korea

Full list of author information is available at the end of the article
} 


\section{Background}

Fatigue generally refers to severe exhaustion during and after daily activities or to a lack of energy. Prolonged and repetitive fatigue seriously influences the quality of life [1]. Fatigue that continues for more than 6 months is defined as chronic fatigue, and chronic fatigue for which no medical explanation exists is classified as chronic fatigue syndrome (CFS) or idiopathic chronic fatigue (ICF). The diagnostic criteria for CFS are as follows: (1) the individual has severe chronic fatigue for 6 or more consecutive months that is not due to ongoing exertion or other medical conditions associated with fatigue; (2) the fatigue significantly interferes with daily activities and work; and (3) the individual concurrently has four or more of eight specific symptoms. These symptoms include the following: (i) postexertional malaise lasting for more than $24 \mathrm{~h}$; (ii) unrefreshing sleep; (iii) significant impairment of shortterm memory or concentration; (iv) muscle pain; (v) multijoint pain without swelling or redness; (vi) headaches of a new type, pattern, or severity; (vii) tender cervical or axillary lymph nodes; and (viii) a sore throat that is frequent or recurring. Information about CFS can be found at http://www.cdc.gov/cfs/case-definition/index.html. Cases that cannot be categorized according to the above diagnostic criteria are included under ICF $[2,3]$. The worldwide prevalence of ICF and CFS is approximately $10 \%$ and $1 \%$, respectively [4]. The causes of CFS and ICF are not clearly known. Immune regulatory dysfunction, various inflammatory conditions, neuroendocrine dysfunction, central nervous system impairments, and stress are the hypothesized causes of them. There is no definitive cure for CFS and ICF. Therefore, patients with CFS and ICF are interested in Oriental medicine or complementary and alternative medicine, and various alternative approaches have been used to treat these patients $[3,5,6]$. For this reason, the effectiveness of complementary and alternative treatments should be verified.

Acupuncture and moxibustion are among the most widely used treatments in Oriental medicine. Thus far, some systematic reviews have reported favorable outcomes regarding the effects of acupuncture and moxibustion on CFS. However, the overall finding of these reviews is that firm evidence for the effects of acupuncture and moxibustion is lacking because of the poor methodological quality of the studies included [7-11]. In this trial, we evaluated the effectiveness of two acupuncture methods: (1) body acupuncture, a traditional acupuncture method widely used for many years in China, Korea, and Japan, in which treatment is applied to the whole body, and (2) Sa-am acupuncture, a Korean traditional acupuncture method developed by Sa-am in the Chosun Dynasty, in which the five-phase theory and mother-child reinforcementreduction principle are applied to the selection of points and needling manipulation [12-14]. This study aimed to evaluate the overall effects of acupuncture treatment by comparing outcomes in a treatment group receiving body acupuncture or Sa-am acupuncture with outcomes in a control group receiving usual care.

\section{Methods}

This was a three-arm, randomized, nonblinded, controlled, and parallel-designed trial. This trial was conducted in four clinical research centers of South Korea from August 2012 to May 2013. The participants were recruited by each center (Gwangju, Seoul, Jecheon, and Daejeon) through advertisements in local newspapers, the websites of local universities, and posters displayed in hospitals. Details of the study design are available in a previous publication [15]. The flow diagram and CONSORT (Consolidated Standards of Reporting Trials) checklist are provided in Additional files 1 and 2.

\section{Ethical considerations}

This study was approved by the institutional review boards of the Gwangju Oriental Hospital of Dongshin University, Kyung Hee University Hospital at Gangdong, Jecheon Oriental Hospital of Semyung University, and Daejeon Oriental Hospital of Daejeon University. All participants provided written informed consent. The study was conducted according to the principles of the Declaration of Helsinki [16].

\section{Inclusion criteria}

The inclusion criteria for the study were as follows $[2,17]$ :

1. Adult men and women aged 19 to 65 years.

2. Presenting major symptoms of continuous or recurrent fatigue due to unknown causes of at least 6 months' duration.

3. Absence of abnormal findings in the following: blood pressure (BP) test, blood tests (hemoglobin $(\mathrm{Hb})$, hematocrit (Hct), white blood cells (WBC), and glucose), biochemical marker tests (aspartate aminotransferase (AST), alanine aminotransferase (ALT), and creatinine), serum electrolyte tests ( $\operatorname{sodium}(\mathrm{Na})$, potassium $(\mathrm{K})$, and chloride $(\mathrm{Cl})$ ), thyroid function tests (TFTs) (thyroid stimulating hormone (TSH) and free thyroxine (FT4)), pregnancy test for women of child-bearing age, chest radiography (CXR), and electrocardiography (ECG).

Test findings were considered "abnormal" with reference to the following values:

a. BP: Diastolic BP $\geq 90 \mathrm{mmHg}$ (average of 2 measurements, each taken at an interval of at least 2 min after a rest period of at least $5 \mathrm{~min}$ in a sitting position). 
b. Blood tests: $\mathrm{Hb}<13$ or $<11.5 \mathrm{~g} / \mathrm{dL}$ and Hct $<38 \%$ or $<36 \%$, for males and non-pregnant females, respectively; $\mathrm{WBC} \geq 11,000 / \mathrm{mm}^{3}$ and random plasma glucose $\geq 200 \mathrm{mg} / \mathrm{dL}$ for all participants.

c. Biochemical marker tests: AST or ALT $\geq 50 \mathrm{IU} / \mathrm{L}$; creatinine $\geq 1.5 \mathrm{mg} / \mathrm{dL}$.

d. Electrolyte tests: $\mathrm{Na}<135$ or $\geq 145 \mathrm{mmol} / \mathrm{L} ; \mathrm{K}<3.5$ or $\geq 5.5 \mathrm{mmol} / \mathrm{L} ; \mathrm{Cl}<97$ or $\geq 110 \mathrm{mmol} / \mathrm{L}$.

e. TFTs: TSH $<0.35$ or $\geq 5.50 \mathrm{mIU} / \mathrm{mL}$; FT $4<0.89$ or $\geq 1.76 \mathrm{ng} / \mathrm{dL}$.

f. Pregnancy test: Urine sample positive for human chorionic gonadotropin (hCG).

g. CXR: Active pulmonary tuberculous lesions.

h. ECG: Indications of heart disease requiring treatment, such as arrhythmia, ischemic heart disease, or cardiomegaly.

4. Numeric Rating Scale (NRS) [18] score $\geq 4$ during the 7 days preceding the screening tests.

5. Providing written informed consent after being informed of the objectives and particularities of the clinical trial, and agreeing to participate in the trial.

\section{Exclusion criteria}

The exclusion criteria for the study were as follows $[2,17]$ :

1. History of chronic fatigue or a present illness that might trigger chronic fatigue, including (but not limited to) the following disorders:

a. Organic causes: Acute or chronic liver diseases (hepatitis, liver cirrhosis), anemia, tuberculosis, chronic lung diseases, cardiovascular diseases (heart failure, hypertension), endocrine/metabolic diseases (diabetes, hyperthyroidism, hypothyroidism, severe obesity with a body mass index (BMI) of $\geq 35 \mathrm{~kg}$ / $\mathrm{m}^{2}$ ), autoimmune diseases (rheumatoid arthritis, systemic lupus erythematosus, multiple sclerosis), malignant tumors, or infectious diseases.

b. Psychosocial causes: Major depression, anxiety neurosis, a recent severe stress, schizophrenia, alcoholism, or an eating disorder (anorexia nervosa, bulimia nervosa).

2. Medication with the following drugs during the preceding 2 weeks: Antihypertensive drugs, antidepressants, antianxiety agents, sleeping pills, or antihistamines.

3. Female participants who were pregnant, breastfeeding, or planning for pregnancy.

4. Participating in other clinical trials.

5. Undertaking excessive workloads (for example, undertaking multiple jobs).
6. History of hypersensitivity reaction to acupuncture treatment.

7. Residents of collective dwelling facilities, such as social welfare institutions.

8. Failure to provide written informed consent.

9. Considered unfit for the trial by the principal investigator because of other reasons.

\section{Randomization}

A statistician used a computer program (Strategic Applications Software (SAS), version 9.1.3 (SAS Institute Inc., Cary, NC, USA) to generate the random allocation sequence. We used a stratified block randomization scheme with four institutions chosen as strata. The generated sequences were sealed in opaque envelopes and delivered to each center, where they were stored in double-locked cabinets. Randomization was conducted for participants who satisfied all selection criteria. The researcher opened the random allocation envelopes in order in front of the participants and assigned them to 1 of 3 groups. The clinical research coordinator allotted participant identification codes and recorded them in the case report forms. The opened envelopes were stored separately in double-locked cabinets.

\section{Blinding}

This was a nonblinded study.

\section{Interventions}

The eligible participants were allocated randomly into three groups, including Group A, Group B, or Group C. In each group, participants received their allocated treatment. Participants in treatment group received 10 sessions of acupuncture treatment for 4 weeks, 2 to 3 times a week, using stainless steel disposable needles (0.25$\mathrm{mm}$ diameter, 30-mm length; Dongbang Acupuncture Inc., South Korea). Participants were informed about the body acupuncture and Sa-am acupuncture used in this study as follows: "In this study, two types of acupuncture will be used. One type will be body acupuncture, which is based on traditional Chinese medicine (TCM). The other type will be Sa-am acupuncture, a traditional Korean acupuncture method." The practitioners, doctors of Korean medicine having more than 3 years of practical experience, participated in a training session to ensure that treatments were administered consistently. The practitioners were allowed to communicate with the participants about treatment, lifestyle, etc.

\section{Group A (body acupuncture plus usual care group)}

The participants in Group A were treated in the supine position at the acupoints described below. The acupoints were selected based on published literature and textbooks [12, 19]: 
1. GV20 (forward horizontal needling up to a depth of 0.5 to 1.5 cun).

2. Bilateral GB20 (perpendicular needling toward the opposite-side eye up to a depth of 0.3 to 1.0 cun).

3. Bilateral BL11, BL13, BL15, BL18, BL20, and BL23 (downward oblique or horizontal needling up to a depth of 0.5 to 1.0 cun).

All needles were retained for 15 min without twirling. In TCM, the qi moves through the meridians at a rate of 6 cun per respiratory cycle. The length of the meridians and collaterals equals 1620 cun. Thus, 270 (1620/6) respiratory cycles are required for $q i$ to make one cycle through the body. If an adult breathes 18 times per min, $15 \mathrm{~min}(270 / 18)$ are needed for $q i$ to make one cycle [20]. Therefore, the needle retaining time was $15 \mathrm{~min}$.

\section{Group B (Sa-am acupuncture plus usual care group)}

The participants assigned to Group B received treatment at the following acupoints while sitting on a chair. The acupoints were selected based on a published literature [13]:

1. LU8 (downward oblique needling up to a depth of 0.5 to 0.7 cun)

2. SP3 (upward oblique needling up to a depth of 0.3 to 0.5 cun)

3. HT8 (downward oblique needling up to a depth of 0.3 to 0.5 cun)

4. BL15 (upward oblique or horizontal needling up to a depth of 0.5 to 1.0 cun)

5. CV6 (downward oblique needling up to a depth of 0.5 to 1.0 cun).

All needles were retained for $15 \mathrm{~min}$ after being twirled nine times (at LU8, SP3, and HT8) or six times (at BL15 and CV6).

\section{Group C (usual care alone group)}

The participants randomized to Group $C$ did not receive acupuncture treatment; however, they did receive the necessary usual care $[21,22]$.

\section{Concomitant treatments}

In all groups, usual care consisted of the use of any form of concomitant treatment, including Oriental medicine treatments (for example, acupuncture for conditions other than chronic fatigue, moxibustion, herbal medicine), Western medicine (for example, conventional medication, injections), or self-care (for example, dietary supplements, exercise). All groups were provided with educational materials about chronic fatigue.

\section{Primary outcome}

The Fatigue Severity Scale (FSS) score at 5 weeks after randomization was used as the primary endpoint. The FSS included nine questions, scored on a scale of 1 to 7 , which were used to evaluate the participant's fatigue level during the previous week $[23,24]$.

\section{Secondary outcomes}

A short form of the Stress Response Inventory (SRI), consisting of 22 questions covering the following three categories, was used to evaluate stress: somatization (nine items), depression (eight items), and anger (five items) [25]. The Beck Depression Inventory (BDI) consisted of 21 questions assessing the cognitive, emotional, motivational, and somatic symptoms of depression. Each item was scored on a 4-point scale from 0 to 3 [26]. The NRS used a horizontal straight line with numbers from 0 to 10 and indications of "no fatigue" on the far left and "most severe fatigue experienced" on the far right end. The participants were asked to select the number that represented the level of their own fatigue [18]. The EuroQol-5 Dimension (EQ-5D) tool assessed healthrelated quality of life. It consisted of the EQ-Visual Analogue Scale (VAS), which scored the participant's health condition on a straight line marked from 0 to 100 , and the EQ-5D descriptive system, which inquired about five dimensions of the participant's current health status. Information about the EQ-5D can be found at http://www.euroqol.org/.

\section{Other assessments}

We assessed the treatment expectancy survey [27] completed by each participant, and confirmed that the Centers for Disease Control and Prevention (CDC)'s diagnostic criteria for CFS [2] was fulfilled.

\section{Adverse events}

Practitioners recorded all unexpected and unintended responses as reported by each participant on an adverse event report form. The relationship between acupuncture treatment and adverse events was graded from 1 to $6(1=$ definitely related, $2=$ probably related, $3=$ possibly related, $4=$ probably not related, $5=$ definitely not related, and $6=$ unknown).

\section{Statistical analysis}

In order to have a $90 \%$ chance of detecting a mean difference of 0.915 in the primary outcome at a significance level of $5 \%, 40$ patients were required per group. Based on the effect size described in a previous pilot study [12], we assumed a mean change of 0.9632 in the treatment group and 0.0482 in the control group, with a common standard deviation of 1.1479. Allowing for a 20 $\%$ dropout rate, 50 patients were required per group 
(150 in total). Details of the sample size calculation are available in a previous publication [15]. A computer program (SAS, version 9.1.3) was used to perform the analysis. The hypothesis of this study was as follows: $H_{0}$ : $\mu_{1}=\mu_{3}, \mu_{2}=\mu_{3}, H_{1}:$ not $H_{0}\left(\mu_{1}\right.$, FSS mean score for Group A at 5 weeks after randomization; $\mu_{2}$, FSS mean score for Group B at 5 weeks after randomization; and $\mu_{3}$, FSS mean score for Group $\mathrm{C}$ at 5 weeks after randomization). The analysis set consisted of a full analysis set (FAS; that is, the set of participants who were as close as possible to the intention-to-treat (ITT) principle) and a per-protocol set (PPS; that is, the set of participants who were more compliant with the protocol) [28]. The FAS was used for the main analysis.

A mixed model for repeated measures (MMRM) method was used to determine the differences between the experimental groups and the control group; the treatment, visit time, and institution were the fixed factors, and the participant was the random factor [29]. Additionally, an analysis of covariance (ANCOVA) was performed by replacing the missing data using the last observation carried forward (LOCF) method, and the results were compared with the results of the MMRM method. In ANCOVA, the primary and secondary outcomes at 5 and 13 weeks after randomization were the dependent variables, the baseline value was the covariate, and the group and the institution were the fixed factors. The analyses were performed at the $5 \%$ significance level. When the ANCOVA produced significant results, a posthoc analysis was conducted to determine which groups showed differences. For the socio-demographic characteristics and treatment expectancy, the mean and standard deviation were determined from the continuous data, and the frequency and percentage were determined from the categorical data. The subgroup analysis of FSS was performed in CFS and ICF groups.

\section{Results}

Flow of participants

We selected 150 participants from 195 screened individuals. Gwangju, Kyung Hee, and Jecheon hospitals recruited 45 participants each, and Daejeon center recruited 15 participants. The selected participants were randomly allocated to a treatment group (Group A or B) or the control group (Group C). Among the participants, three from Group A, two from Group B, and two from Group $C$ dropped out of the study (see Additional file 1). The reasons the participants dropped out of the study are presented in Additional file 1.

\section{Baseline characteristics}

The sociodemographic characteristics of the participants are shown in Table 1. No significant differences were observed between the groups regarding age, sex, height, weight, education level, occupation, marital status, meal regularity, exercise, smoking, and use of alcohol. According to the CFS questionnaire, 63 participants (42\%) could be classified as having CFS. The frequency of participants with CFS was similar within the different groups. The treatment expectancy was significantly different between the treatment groups and the control group (Table 1).

\section{Findings for the primary outcome}

The FSS score from Group A at 5 weeks after randomization was significantly lower than that of Group C (Table 2).

\section{Findings for the secondary outcomes}

The FSS scores at 13 weeks after randomization were not different between the groups. The SRI-short form scores were significantly lower in the treatment groups than in the control group at both 5 and 13 weeks after randomization. Although no difference was observed in the BDI score among the groups at 5 weeks after randomization, the score from Group B was significantly reduced in comparison to Group $\mathrm{C}$ at 13 weeks after randomization. The NRS scores from the treatment groups were significantly reduced compared to those from the control group at both 5 and 13 weeks after randomization. The EQ-5D did not show any differences among the groups at 5 and 13 weeks after randomization (Table 2).

\section{Sensitivity analysis}

After processing the missing values with the LOCF method, the results of the ANCOVA indicated that the scores from the FSS, SRI-short form, BDI, and NRS were significantly reduced in the treatment groups compared to the control group at both 5 and 13 weeks after randomization. The EQ-5D was significantly different between Group A and $\mathrm{C}$ at 5 weeks after randomization and between both treatment groups and the control group at 13 weeks after randomization (Table 3 ).

The results of the PPS analysis were similar to those of the FAS. However, the SRI-short form score at 13 weeks after randomization was not significantly different between Group A and C (Table 4).

The FSS score at 5 weeks after randomization, performed on the FAS after correcting for the treatment expectancy in each group, showed a significant difference between Group A and C $(P=0.028)$. A significant difference was not observed between Group B and $\mathrm{C}(P=0.129)$.

\section{Subgroup analysis}

The CFS group comprised 21 patients in Group A, 23 in Group B, and 19 in Group C. Furthermore, the ICF 
Table 1 Baseline characteristics of study participants

\begin{tabular}{|c|c|c|c|c|}
\hline Variable & & Group A $(n=49)$ & Group B $(n=51)$ & Group C $(n=50)$ \\
\hline Age, years & & $44.9 \pm 11.4$ & $39.6 \pm 11.5$ & $41.1 \pm 11.9$ \\
\hline Sex, M/F & & $15(30.6) / 34(69.4)$ & $18(35.3) / 33(64.7)$ & $19(38.0) / 31(62.0)$ \\
\hline Height, cm & & $162.2 \pm 6.8$ & $165.3 \pm 8.9$ & $164.4 \pm 8.8$ \\
\hline Weight, kg & & $59.8 \pm 10.8$ & $63.0 \pm 12.5$ & $62.8 \pm 12.4$ \\
\hline \multicolumn{5}{|l|}{ Duration of chronic fatigue } \\
\hline & 6 months to 1 year & $17(34.7)$ & $13(25.5)$ & $18(36.0)$ \\
\hline & 1 year to 5 years & $17(34.7)$ & $23(45.1)$ & $24(48.0)$ \\
\hline & $>5$ years & 15 (30.6) & $15(29.4)$ & $8(16.0)$ \\
\hline \multicolumn{5}{|l|}{ Previous treatment } \\
\hline & Medication & $0(0.0)$ & $2(3.9)$ & $0(0.0)$ \\
\hline & Oriental medicine treatment & $2(4.1)$ & $4(7.8)$ & $1(2.0)$ \\
\hline & Therapeutic exercise & $1(2.0)$ & $3(5.9)$ & $0(0.0)$ \\
\hline & Psychotherapy & $0(0.0)$ & $0(0.0)$ & $0(0.0)$ \\
\hline & Other treatment & $6(12.2)$ & $4(7.8)$ & $5(10.0)$ \\
\hline Chronic fatigue syndrome, M/F & & $3(6.1) / 18(36.7)$ & $6(11.8) / 17(33.3)$ & $6(12.0) / 13(26.0)$ \\
\hline Education $>12$ years & & $28(57.1)$ & $39(76.5)$ & $35(70.0)$ \\
\hline Job & & $36(73.5)$ & $39(76.5)$ & $40(80.0)$ \\
\hline Marriage & & $38(77.6)$ & $32(62.7)$ & $37(74.0)$ \\
\hline Irregular meals & & $18(36.7)$ & $18(35.3)$ & $19(38.0)$ \\
\hline \multicolumn{5}{|l|}{ Exercise } \\
\hline & Yes & $29(59.2)$ & $24(47.1)$ & $23(46.0)$ \\
\hline & Weekly average frequency & $3.4 \pm 2.0$ & $2.8 \pm 1.5$ & $3.4 \pm 1.6$ \\
\hline & Average time per session, h & $1.2 \pm 0.9$ & $1.4 \pm 0.6$ & $1.1 \pm 0.5$ \\
\hline Tobacco use & & $8(16.3)$ & $10(19.6)$ & $8(16.0)$ \\
\hline Alcohol use & & $23(46.9)$ & $21(41.2)$ & $21(42.0)$ \\
\hline \multirow[t]{3}{*}{ Treatment expectancy } & & $6.9 \pm 1.5$ & $6.1 \pm 1.4$ & $4.5 \pm 2.4$ \\
\hline & Yes (6 to 9) & $40(81.6)$ & $37(72.5)$ & $18(36.0)$ \\
\hline & No (1 to 5$)$ & $9(18.4)$ & $14(27.5)$ & $32(64.0)$ \\
\hline
\end{tabular}

Data are presented as either mean \pm SD or number (\%), where appropriate

group comprised 28 patients in Group A, 28 in Group B, and 31 in Group C. Results of MMRM analysis of FSS score in each group and results of the subgrouptreatment effect interaction test are presented in Table 5.

\section{Concomitant treatments}

Usual care methods for fatigue reported during the trial period were nutritional supplements and exercise (working out, walking, riding a stationary bicycle, swimming, and hiking). In terms of individual groups, Group A had two patients who took nutritional supplements, three patients who performed exercise, and one patient who combined nutritional supplements with exercise; whereas Group B had two patients who took nutritional supplements, one patient who received a nutritional supplement injection, and three patients who performed exercise. In Group C, two patients took nutritional supplements and three patients performed exercise. None of the participants in Group A or C received any additional acupuncture during the study period, other than the therapy provided as part of the study. One participant in Group $B$ had received acupuncture for lower back pain, which was unrelated to fatigue.

\section{Adverse events}

A total of 10 adverse events were reported by 10 participants. Of these events, two were determined to be linked either to acupuncture or possibly associated with acupuncture, and the symptom severity was mild in both cases. All participants who experienced adverse events improved during the research period and continued to participate in the experiment. Although two serious adverse events were reported, they were not related to the 
Table 2 Results of mixed model for repeated measures (MMRM) analysis of full analysis set (FAS)

\begin{tabular}{|c|c|c|c|c|c|c|c|c|}
\hline \multirow[t]{2}{*}{ Outcome } & \multirow[t]{2}{*}{ Group } & \multirow{2}{*}{$\begin{array}{l}\text { Baseline } \\
\text { Mean (SD) }\end{array}$} & \multicolumn{3}{|l|}{5 weeks } & \multicolumn{3}{|l|}{13 weeks } \\
\hline & & & Mean (SD) & $P^{a}$ & LSMD $(95 \% \text { Cl) })^{a}$ & $\overline{\text { Mean (SD) }}$ & $P^{a}$ & $\operatorname{LSMD}(95 \% \mathrm{Cl})^{\mathrm{a}}$ \\
\hline \multirow[t]{3}{*}{ FSS } & $A$ & $4.67(1.27)$ & $3.38(1.01)$ & 0.023 & $-0.43(-0.81,-0.05)$ & $3.34(1.17)$ & 0.075 & $-0.36(-0.74,0.03)$ \\
\hline & B & $4.75(1.07)$ & $3.58(1.12)$ & 0.151 & $-0.29(-0.66,0.08)$ & $3.51(1.19)$ & 0.290 & $-0.23(-0.62,0.15)$ \\
\hline & C & $4.48(1.27)$ & $4.47(1.18)$ & - & - & $4.28(1.24)$ & - & - \\
\hline \multirow[t]{3}{*}{ SRI-short form } & A & $62.56(17.95)$ & $42.21(14.18)$ & 0.032 & $-6.01(-11.58,-0.44)$ & $42.28(14.33)$ & 0.037 & $-6.19(-12.06,-0.31)$ \\
\hline & B & $55.67(14.40)$ & $42.10(13.66)$ & $<0.001$ & $-9.33(-14.72,-3.95)$ & $39.81(13.55)$ & $<0.001$ & $-10.80(-16.49,-5.12)$ \\
\hline & C & $60.04(18.16)$ & $56.47(18.03)$ & - & - & $57.41(20.46)$ & - & - \\
\hline \multirow[t]{3}{*}{$\mathrm{BDI}$} & A & $16.41(8.36)$ & $9.02(6.92)$ & 0.252 & $-1.56(-3.94,0.83)$ & $9.63(6.39)$ & 0.231 & $-1.68(-4.16,0.81)$ \\
\hline & B & $15.00(7.67)$ & $8.88(7.66)$ & 0.052 & $-2.34(-4.70,0.02)$ & $7.89(7.15)$ & 0.007 & $-3.25(-5.71,-0.80)$ \\
\hline & C & $15.30(7.05)$ & $13.36(6.39)$ & - & - & $14.28(8.54)$ & - & - \\
\hline \multirow[t]{3}{*}{ NRS } & A & $6.59(1.44)$ & $4.42(1.98)$ & $<0.001$ & $-0.92(-1.45,-0.40)$ & $4.77(2.22)$ & 0.011 & $-0.70(-1.26,-0.14)$ \\
\hline & B & $6.55(1.27)$ & $4.52(1.70)$ & $<0.001$ & $-0.90(-1.42,-0.39)$ & $4.51(1.95)$ & 0.002 & $-0.85(-1.40,-0.29)$ \\
\hline & C & $6.40(1.40)$ & $6.53(1.36)$ & - & - & $6.42(1.69)$ & - & - \\
\hline \multirow[t]{3}{*}{ EQ-5D } & A & $0.84(0.10)$ & $0.91(0.11)$ & 0.909 & $-0.01(-0.04,0.03)$ & $0.92(0.11)$ & 0.844 & $0.01(-0.03,0.04)$ \\
\hline & B & $0.87(0.09)$ & $0.91(0.09)$ & 0.522 & $0.01(-0.02,0.05)$ & $0.94(0.08)$ & 0.074 & $0.03(0.00,0.07)$ \\
\hline & C & $0.88(0.11)$ & $0.88(0.10)$ & - & - & $0.87(0.15)$ & - & - \\
\hline
\end{tabular}

LSMD, least squares mean difference; $\mathrm{Cl}$, confidence interval; FSS, fatigue severity scale; SRI-short form, short form of the stress response inventory; $\mathrm{BDI}$, Beck depression inventory; NRS, numeric rating scale; EQ-5D, EuroQol-5 dimension.

${ }^{\text {a }} P$ value with $95 \% \mathrm{Cl}$, Dunnett's adjustment

acupuncture (that is, surgery for a nasal problem and hospitalization because of a traffic accident) (Table 6).

\section{Discussion}

After 10 treatment sessions in 4 weeks, the mean FSS score in Group A improved significantly compared to
Group C. This result is consistent with findings in a preliminary study on body acupuncture [12]. A previous study reported that the optimal FSS cut-off point to demarcate between fatigue and normal groups was 3.22 (sensitivity $84.1 \%$, specificity $85.7 \%$ ) [24]. Using this cut-off point as the reference standard, the mean post-

Table 3 Results of analysis of covariance (ANCOVA) of full analysis set (FAS)

\begin{tabular}{|c|c|c|c|c|c|c|c|c|}
\hline \multirow[t]{2}{*}{ Outcome } & \multirow[t]{2}{*}{ Group } & \multirow{2}{*}{$\begin{array}{l}\text { Baseline } \\
\text { Mean (SD) }\end{array}$} & \multicolumn{3}{|l|}{5 weeks } & \multicolumn{3}{|l|}{13 weeks } \\
\hline & & & Mean (SD) & $P^{a}$ & LSMD $(95 \% \text { CI) })^{a}$ & $\overline{\text { Mean (SD) }}$ & $P^{a}$ & LSMD $(95 \%$ CI) \\
\hline \multirow[t]{3}{*}{ FSS } & A & $4.67(1.27)$ & $3.39(1.12)$ & $<0.001$ & $-1.14(-1.57,-0.71)$ & $3.32(1.17)$ & $<0.001$ & $-1.01(-1.48,-0.55)$ \\
\hline & B & $4.75(1.07)$ & $3.62(1.13)$ & $<0.001$ & $-0.95(-1.38,-0.52)$ & $3.58(1.20)$ & $<0.001$ & $-0.80(-1.26,-0.34)$ \\
\hline & C & $4.48(1.27)$ & $4.43(1.19)$ & - & - & $4.24(1.25)$ & - & - \\
\hline \multirow[t]{3}{*}{ SRI-short form } & A & $62.56(17.95)$ & $43.07(15.49)$ & $<0.001$ & $-15.24(-21.32,-9.16)$ & $43.61(15.31)$ & $<0.001$ & $-15.01(-21.96,-8.06)$ \\
\hline & B & $55.67(14.40)$ & $42.65(14.24)$ & $<0.001$ & $-11.58(-17.45,-5.72)$ & $40.19(13.57)$ & $<0.001$ & $-14.97(-21.67,-8.26)$ \\
\hline & C & $60.04(18.16)$ & $56.82(18.10)$ & - & - & $57.36(20.30)$ & - & - \\
\hline \multirow[t]{3}{*}{$\mathrm{BDI}$} & A & $16.41(8.36)$ & $9.43(6.98)$ & $<0.001$ & $-5.03(-7.60,-2.47)$ & $10.02(6.40)$ & $<0.001$ & $-4.74(-7.56,-1.91)$ \\
\hline & B & $15.00(7.67)$ & $9.04(7.61)$ & $<0.001$ & $-4.63(-7.17,-2.10)$ & $8.20(7.01)$ & $<0.001$ & $-5.88(-8.67,-3.09)$ \\
\hline & C & $15.30(7.05)$ & $13.84(6.80)$ & - & - & $14.22(8.32)$ & - & - \\
\hline \multirow[t]{3}{*}{ NRS } & A & $6.59(1.44)$ & $4.56(2.09)$ & $<0.001$ & $-1.96(-2.72,-1.20)$ & $4.78(2.32)$ & $<0.001$ & $-1.64(-2.51,-0.78)$ \\
\hline & B & $6.55(1.27)$ & $4.58(1.70)$ & $<0.001$ & $-1.93(-2.68,-1.18)$ & 4.59 (1.92) & $<0.001$ & $-1.81(-2.67,-0.96)$ \\
\hline & C & $6.40(1.40)$ & $6.46(1.39)$ & - & - & $6.34(1.70)$ & - & - \\
\hline \multirow[t]{3}{*}{ EQ-5D } & A & $0.84(0.10)$ & $0.96(0.11)$ & 0.025 & $0.04(0.00,0.08)$ & $0.91(0.11)$ & 0.008 & $0.06(0.01,0.11)$ \\
\hline & B & $0.87(0.09)$ & $0.91(0.09)$ & 0.107 & $0.03(-0.01,0.07)$ & $0.93(0.09)$ & 0.005 & $0.06(0.02,0.11)$ \\
\hline & C & $0.88(0.11)$ & $0.88(0.09)$ & - & - & $0.87(0.15)$ & - & - \\
\hline
\end{tabular}

LSMD, least squares mean difference; $\mathrm{Cl}$, confidence interval; FSS, fatigue severity scale; SRI-short form, short form of the stress response inventory; $\mathrm{BDI}$, Beck depression inventory; NRS, numeric rating scale; EQ-5D, EuroQol-5 dimension

${ }^{a} P$ value with $95 \% \mathrm{Cl}$, Dunnett's adjustment 
Table 4 Results of mixed model for repeated measures (MMRM) analysis of per-protocol set (PPS)

\begin{tabular}{|c|c|c|c|c|c|c|c|c|}
\hline \multirow[t]{2}{*}{ Outcome } & \multirow[t]{2}{*}{ Group } & \multirow{2}{*}{$\begin{array}{l}\text { Baseline } \\
\text { Mean (SD) }\end{array}$} & \multicolumn{3}{|l|}{5 weeks } & \multicolumn{3}{|l|}{13 weeks } \\
\hline & & & Mean (SD) & $P^{a}$ & LSMD $(95 \% \text { Cl) })^{a}$ & $\overline{\text { Mean (SD) }}$ & $P^{a}$ & $\operatorname{LSMD}(95 \% \mathrm{Cl})^{\mathrm{a}}$ \\
\hline \multirow[t]{3}{*}{ FSS } & A & $4.70(1.22)$ & $3.38(1.10)$ & 0.017 & $-0.45(-0.84,-0.07)$ & $3.34(1.17)$ & 0.074 & $-0.36(-0.75,0.03)$ \\
\hline & B & $4.74(1.09)$ & $3.58(1.12)$ & 0.090 & $-0.33(-0.71,0.04)$ & $3.51(1.19)$ & 0.230 & $-0.26(-0.64,0.12)$ \\
\hline & C & $4.52(1.26)$ & $4.47(1.18)$ & - & - & $4.24(1.22)$ & - & - \\
\hline \multirow[t]{3}{*}{ SRI-short form } & $A$ & $62.59(17.77)$ & $42.21(14.18)$ & 0.039 & $-5.92(-11.58,-0.27)$ & $42.28(14.33)$ & 0.063 & $-5.69(-11.64,0.26)$ \\
\hline & B & $55.50(14.23)$ & $42.10(13.66)$ & $<0.001$ & $-9.22(-14.69,-3.76)$ & $39.81(13.55)$ & $<0.001$ & $-10.34(-16.10,-4.58)$ \\
\hline & C & $59.26(18.34)$ & $56.47(18.03)$ & - & - & $56.67(20.11)$ & - & - \\
\hline \multirow[t]{3}{*}{$\mathrm{BDI}$} & A & $16.59(8.60)$ & $9.02(6.92)$ & 0.338 & $-1.39(-3.82,1.04)$ & $9.64(6.39)$ & 0.386 & $-1.35(-3.88,1.18)$ \\
\hline & B & $15.12(7.67)$ & $8.88(7.66)$ & 0.086 & $-2.14(-4.54,0.25)$ & $7.89(7.15)$ & 0.018 & $-2.92(-5.41,-0.43)$ \\
\hline & C & $14.91(6.78)$ & $13.36(6.39)$ & - & - & $14.00(8.42)$ & - & - \\
\hline \multirow[t]{3}{*}{ NRS } & A & $6.57(1.42)$ & $4.42(1.98)$ & $<0.001$ & $-1.01(-1.54,-0.48)$ & $4.77(2.22)$ & 0.006 & $-0.76(-1.33,-0.20)$ \\
\hline & B & $6.53(1.28)$ & $4.52(1.70)$ & $<0.001$ & $-0.98(-1.50,-0.46)$ & $4.51(1.95)$ & $<0.001$ & $-0.90(-1.46,-0.34)$ \\
\hline & C & $6.47(1.38)$ & $6.53(1.36)$ & - & - & $6.38(1.69)$ & - & - \\
\hline \multirow[t]{3}{*}{ EQ-5D } & $A$ & $0.84(0.10)$ & $0.91(0.11)$ & 0.948 & $0.00(-0.04,0.03)$ & $0.92(0.11)$ & 0.785 & $0.01(-0.03,0.05)$ \\
\hline & B & $0.87(0.10)$ & $0.91(0.09)$ & 0.494 & $0.01(-0.02,0.05)$ & $0.94(0.08)$ & 0.067 & $0.03(0.00,0.07)$ \\
\hline & C & $0.88(0.11)$ & $0.88(0.10)$ & - & - & $0.87(0.15)$ & - & - \\
\hline
\end{tabular}

LSMD, least squares mean difference; $\mathrm{Cl}$, confidence interval; FSS, fatigue severity scale; SRI-short form, short form of the stress response inventory; $\mathrm{BDI}$, Beck depression inventory; NRS, numeric rating scale; EQ-5D, EuroQol-5 dimension

${ }^{a} P$ value with $95 \% \mathrm{Cl}$, Dunnett's adjustment

treatment FSS score for Group A (3.38) can be considered a near-normal value. Group B demonstrated improvement after 10 treatment sessions in 4 weeks, with an FSS score of 3.58. However, the change was not statistically significant and contradicted findings in a preliminary study of Sa-am acupuncture [13]. The discrepancy between the results of the present and preliminary study may be due to the higher proportion of participants (approximately 28 \%) with fatigue morbidity lasting less than 6 months in the preliminary study. The shorter duration of morbidity in the preliminary study likely eased the task of alleviating symptoms compared to the longer duration in the present study. Additionally, in the preliminary study, a sham acupuncture group was used as a control, and the Multiple Fatigue Scale (MFS) was used for assessment, neither of which was employed in this study. The MFS is a reconstituted fatigue assessment tool developed in 2000 and based on the Fatigue Assessment Inventory [30], and its correlation with the FSS has not yet been determined. These differences in the duration of symptoms, control groups, and assessment tools likely contributed to different results between this study and the preliminary study. When a significant result is required for only one of multiple primary endpoints in order to consider a trial positive, each endpoint must be tested with a significance level that has been corrected for multiplicity [31]. For the two primary endpoint tests in this study, we corrected type I errors using Dunnett's method [32], and based on the results of the hypothesis tests, one of two null hypotheses was rejected. Therefore, the results of our trial can be considered positive.

In the auxiliary parameters, the FSS score in Group A decreased to 3.34 at 13 weeks after randomization; however, this trend was not significantly different from results

Table 5 Results of the mixed model for repeated measures (MMRM) analysis of fatigue severity scale (FSS) score in the subgroups

\begin{tabular}{|c|c|c|c|c|c|c|c|c|c|c|}
\hline \multicolumn{2}{|l|}{ Subgroup } & \multirow{2}{*}{$\begin{array}{l}\text { Baseline } \\
\text { Mean (SD) }\end{array}$} & \multicolumn{4}{|l|}{5 weeks } & \multicolumn{4}{|l|}{13 weeks } \\
\hline & & & Mean (SD) & $p^{a}$ & LSMD $(95 \% C l)^{a}$ & Interaction $^{\mathrm{b}}$ & Mean (SD) & $p^{a}$ & LSMD $(95 \% C l)^{a}$ & Interaction $^{\mathrm{b}}$ \\
\hline \multirow[t]{3}{*}{ CFS $(n=63)$} & $A$ & $5.50(0.94)$ & $3.62(1.22)$ & 0.993 & $0.02(-0.51,0.56)$ & $0.585^{c}$ & $3.43(1.21)$ & 0.924 & $-0.08(-0.64,0.48)$ & $0.854^{c}$ \\
\hline & B & $5.06(0.87)$ & $3.75(1.00)$ & 0.768 & $-0.14(-0.66,0.38)$ & & $3.95(1.05)$ & 0.901 & $-0.09(-0.64,0.46)$ & \\
\hline & C & $4.74(0.81)$ & $4.39(0.97)$ & - & - & & $4.44(1.23)$ & - & - & \\
\hline \multirow[t]{3}{*}{$\operatorname{ICF}(\mathrm{n}=87)$} & A & $4.06(1.14)$ & $3.21(0.99)$ & 0.008 & $-0.68(-1.20,-0.16)$ & & $3.27(1.15)$ & 0.078 & $-0.48(-1.01,0.04)$ & \\
\hline & $B$ & $4.50(1.16)$ & $3.44(1.21)$ & 0.188 & $-0.37(-0.89,0.14)$ & & $3.16(1.20)$ & 0.235 & $-0.35(-0.87,0.17)$ & \\
\hline & C & $4.32(1.47)$ & $4.51(1.29)$ & - & - & & $4.19(1.25)$ & - & - & \\
\hline
\end{tabular}


Table 6 Adverse events

\begin{tabular}{|c|c|c|c|c|c|c|c|c|}
\hline Symptom & $\begin{array}{l}\text { Patient } \\
\text { numbers }\end{array}$ & $\begin{array}{l}\text { Start } \\
\text { date }\end{array}$ & End date & Intensity & $\begin{array}{l}\text { Causal } \\
\text { relationship }\end{array}$ & $\begin{array}{l}\text { Action related to the } \\
\text { intervention }\end{array}$ & Treatment & Outcome \\
\hline $\begin{array}{l}\text { Redness and itching } \\
\text { around CV6 }\end{array}$ & 1 & 20120918 & 20120920 & Mild & Probably & No change & None & Cured \\
\hline $\begin{array}{l}\text { Right thumb } \\
\text { numbness, pain }\end{array}$ & 1 & 20120913 & 20121120 & Mild & Possibly & No change & None & Improved \\
\hline Nasal obstruction & 1 & 20121031 & 20121031 & $\begin{array}{l}\text { Serious } \\
\text { adverse event }\end{array}$ & $\begin{array}{l}\text { Definitely } \\
\text { not }\end{array}$ & No change & $\begin{array}{l}\text { Medication, non-drug } \\
\text { treatment }\end{array}$ & Cured \\
\hline $\begin{array}{l}\text { Left buttock pain } \\
\text { (traffic accident) }\end{array}$ & 1 & 20120928 & 20121006 & $\begin{array}{l}\text { Serious } \\
\text { adverse event }\end{array}$ & $\begin{array}{l}\text { Definitely } \\
\text { not }\end{array}$ & No change & Medication & Improved \\
\hline Coccyx fracture & 1 & 20121213 & Unknown & Moderate & $\begin{array}{l}\text { Definitely } \\
\text { not }\end{array}$ & No change & Medication & Improved \\
\hline Right shoulder pain & 1 & 20121105 & 20121130 & Mild & $\begin{array}{l}\text { Definitely } \\
\text { not }\end{array}$ & No change & Medication & Improved \\
\hline $\begin{array}{l}\text { Left second finger } \\
\text { pain }\end{array}$ & 1 & 20121101 & 20121203 & Mild & $\begin{array}{l}\text { Definitely } \\
\text { not }\end{array}$ & No change & Medication & Improved \\
\hline \multirow[t]{3}{*}{$\begin{array}{l}\text { Upper respiratory } \\
\text { infection }\end{array}$} & 3 & 20121123 & 20121201 & Mild & $\begin{array}{l}\text { Probably } \\
\text { not }\end{array}$ & No change & None & Improved \\
\hline & & 20121127 & 20121203 & Mild & $\begin{array}{l}\text { Definitely } \\
\text { not }\end{array}$ & No change & None & Improved \\
\hline & & 20121021 & 20121027 & Moderate & $\begin{array}{l}\text { Definitely } \\
\text { not }\end{array}$ & No change & None & Improved \\
\hline
\end{tabular}

in the control group. Therefore, the effects of body acupuncture therapy appears to have been short-term. Group A showed significant improvements in both the SRI-short form and NRS scores at 5 and 13 weeks after randomization. Group B showed significant improvements in the SRI-short form and NRS scores at 5 and 13 weeks after randomization, and in the BDI score at 13 weeks after randomization. Both treatment groups showed improvements in stress or depression, symptoms that are closely associated with fatigue, suggesting that body acupuncture and Sa-am acupuncture may positively influence fatigue in multidimensional or comprehensive aspects. Both Groups A and B showed a significantly lower NRS score, suggesting that body acupuncture and Sa-am acupuncture may positively influence the intensity of fatigue. Reportedly, the FSS assesses the impact rather than the intensity of fatigue $[33,34]$. Thus, the NRS may measure the intensity of fatigue more efficiently than the FSS. Based on the analysis of the SRI-short form, NRS, and BDI results, it was difficult to conclude that Sa-am acupuncture had no effect to improve fatigue. Though it was beyond the scope of this study, the EQ-5D-assisted measurements of the health-related quality of life were performed as a costutility analysis of an economic evaluation. The results showed that neither treatment group exhibited significant improvements at 5 and 13 weeks after randomization. This might be because the treated participants did not experience any changes in their quality of life or because the EQ-5D was a generic instrument that was not diseasespecific and, thus, was not sensitive enough to respond to changes in fatigue severity [35]. Similar to the primary evaluation variable analysis, the results obtained using Dunnett's test were presented for the secondary evaluation variables. However, type I errors were not corrected for all the comparisons in this trial. All comparisons, except for the primary analysis designated before beginning the study, pose a risk of increased type I errors due to multiple tests. The results of secondary analyses do not provide answers to the research question; they should be used for obtaining useful information from the trial [31].

The natural history of chronic fatigue/CFS is still of concern: many patients reported either residual symptoms or disability at follow-up and a progression or worsening of symptoms was seen in some. [36]. Therefore, it was difficult to specify a standard for recurrent fatigue in the inclusion criteria. To date, no specific diagnostic test has been established for CFS [37]; as a result, CFS is diagnosed on the basis of exclusion, subjective clinical interpretation, and patient self-report [38]. In this trial, we included participants by relying on exclusion based on laboratory tests and case definitions. In addition, we used subjective questionnaires when evaluating the outcomes.

At the beginning of this study, we had planned to process the missing data by the LOCF method. However, since the LOCF method can present problems, such as introducing a bias from incorrect estimation of the variance in effect estimates, the main analysis method was changed to the MMRM approach, and the LOCF ANCOVA method was added as a secondary analysis. The results from the two methods were then compared, and we found that the LOCF ANCOVA underestimated 
the $P$ value. This is consistent with the results of previous studies noting that conservative behavior of LOCF is not guaranteed. Therefore, care should be taken when using the LOCF method for analysis of clinical trial data [39, 40]. Because disease severity is different for CFS and ICF, post-hoc subgroup analysis was performed to investigate differences in the therapeutic effects in each group (CFS or ICF). The interpretation of subgroup analysis does not ascertain whether the therapeutic effects exist in each subgroup, but tries to ascertain whether the interaction between the subgroups and the therapeutic effects is significant. Since there was no interaction between the subgroups and therapeutic effects in the present study, acupuncture appears to have been equally effective in all the subgroups. However, because this was an exploratory subgroup analysis, the reproducibility needs to be confirmed in other trials [41].

Although there are no traditional Chinese medical terms that describe CFS and ICF, the concepts may be similar to consumptive disease (a general term for chronic deficiency diseases due to consumption of yin, yang, qi, and blood) or fatigue due to overexertion (a diseased state caused by overexertion, manifested as fatigue, lassitude, shortness of breath upon exertion, and spontaneous sweating) [14, 42]. It was reported that the visceral pattern identification of CFS/ICF can be classified into spleen-lung $q i$ deficiency, spleen-kidney yang deficiency, heart-spleen blood deficiency, and liver-kidney yin deficiency [42, 43]. However, we did not apply pattern identification because we could not find a tool that was sufficiently validated.

In this trial, the acupoints used in Group A were selected to relieve the mind, clear the spirit, tranquilize the body to free it from tension, and regulate the functions of the internal organs [12]. Sa-am acupuncture is largely divided into fixed pattern, transformed pattern, and experienced prescription. Transformed pattern (LU8, SP3, HT8) and experienced prescription (BL15, CV6) were applied to the participants in Group B $[44,45]$. In TCM, needle therapy harmonizes the $q i$ that flows through the meridians of the body. Harmonizing the $q i$ is effective when the $q i$ is obtained in response to acupuncture needling (de qi, needling sensation). Among the methods for harmonizing the $q i$, cui qi or hastening the $q i$, is a way of facilitating de $q i$ if $q i$ does not come when it should, whereas xing $q i$ or moving the $q i$, can aid in transferring the needling response to the disease location. Bu xie, or supplementation and draining, is a method of reinforcing what is lacking and reducing what is excessive. Manual acupuncture can be categorized as basic, assistant, buxie, as well as comprehensive bu xie manual. The basic manual method is a simple technique that involves induction of cui qi and xing $q i$, as well as $b u x i e$. This technique includes twirling and needle retention. Twirling involves turning the needle to the left and to the right, whereas needle retention is designed to maintain the needle at a specific point for a period of time. These basic manual methods can be applied as basic bu xie manual acupuncture techniques. Among the basic bu xie manual methods, the directional method includes ' $b u$ ', which is supplementation via insertion of the needle in the direction of the needled channel flow and 'xie', which is draining via insertion of the needle in the direction opposite to the channel flow. The nine-six method, which is another basic bu xie manual method, distinguishes the number of movements involved in twirling or needle retention, where the number 9 represents ' $b u$ ' and 6 represents ' $x i e$ ' $[14,46,47]$. If one locates the point accurately, $q i$ may be obtained immediately after one inserts the needle to the right depth in the right direction [47]. In the present study, needling was performed in Group A by obtaining accurate acupoints and a suitable depth of needling, along with proper perception by the acupuncturist. Therefore, although twirling and $b u$ xie were not performed in Group A, it is believed that the $q i$ was correctly obtained. In the original literature on Sa-am acupuncture, detailed descriptions of bu xie were not provided, presumably because it was a general contemporary bu xie method or because it was a method based on the traditional bu xie method from Neijing [48]. In Group B, needling was performed by the manual methods (twirling, needle retention, and directional methods) used in the preliminary study [13], as well as the nine-six method added in this trial.

This study has three primary strengths. First, the external validity of the study was improved by performing a pragmatic clinical trial (PCT). Clinical trials can have either an explanatory or a pragmatic design. An explanatory clinical trial (ECT) evaluates what interventions work in ideal (controlled) situations, whereas a PCT evaluates whether interventions work in everyday clinical practice settings. Since PCTs reflect clinical practice, the patient population range is broader than in ECTs, and PCTs often allow for individualized treatments for patients [21, 22]. In previous studies, acupuncture therapy for chronic fatigue has often been limited to CFS [7-11]. In this study, we used usual care as the control and we included ICF patients, as well as CFS patients, in the subject population. Moreover, the study was intended to better reflect real clinical situations through its multi-institutional clinical trial design. However, we did not evaluate the long-term effects of the treatment investigated, unlike general PCTs. In addition, we used a standardized treatment and have therefore studied the effectiveness of the selected points. Second, the results from the FAS and PPS were presented together. A FAS is the collection of participants who most closely fit the ITT principle of the clinical trial, and which minimizes the number of participants excluded from analysis. A PPS is a subset of the FAS and consists of fully compliant participants. In this study, since the results for 
the two sets were similar, confidence in the trial outcomes was increased [28]. Third, adverse events were reported in detail. We described the symptoms and signs, dates of presentation and disappearance, causal relationships with test interventions, acupuncture-related actions, treatments, and results. During the clinical trial period, 2 of the 150 participants $(1.3 \%)$ showed acupuncture-related adverse events. The safety of acupuncture therapy has been reported previously [49-51] and is confirmed herein.

This study has some weaknesses. First, the effectiveness of acupuncture in this study included both specific effects and context effects (expectation, patient-practitioner relationship, etc.) [52]. Although the results compensated for treatment expectations, which are presented in the results section as a reference, the specific effects of acupuncture could not be isolated owing to the study design. It was reported that the identities of CFS patients are challenged when the legitimacy of their illness is questioned, and doctors can support patients in coping with their disease by encouraging them instead of casting suspicion upon them [53]. In the present study, free communication between the patients and the acupuncturist was allowed. However, acupuncturists did not actively provide attention, advice, or empathy. Therefore, it is thought that the patientpractitioner relationship did not significantly impact the treatment effects. Second, there is lower internal validity owing to the allowance of individualized usual care (except acupuncture therapy for chronic fatigue). Although the percentage of participants who reported using usual care during the trial period was not high (Group A: $12 \%$; B: $12 \%$; and C: $10 \%$ ), its effect on the study's outcomes could not be evaluated. Third, fatigue could not be evaluated from multidimensional aspects. The FSS is one of the best known and most used fatigue scales [33, 54]. Since the reliability and validity of the Korean-language version of the FSS has been proven [24], it was used as the primary evaluation instrument for this study. Although the FSS consists of multiple items, it is still a one-dimensional scale and, therefore, is not sufficient for the evaluation of complex aspects of fatigue such as its physical, psychological, and social dimension features [55]. However, there is no instrument among the multidimensional fatigue scales that is used globally and that has been translated into Korean and tested for reliability and validity. In consequence, we did not use a multidimensional instrument.

Although this study did not conclusively demonstrate the effectiveness of acupuncture for CFS and ICF, the results could serve as a reference in the treatment of patients with chronic fatigue of unknown causes.

\section{Conclusions}

The results of this study suggest that 4 weeks of body acupuncture therapy added to usual care can improve fatigue symptoms of CFS and ICF patients.

\section{Additional files}

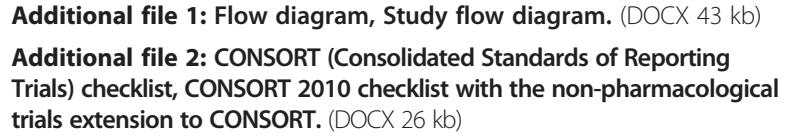

\section{Abbreviations}

ALT: alanine aminotransferase; ANCOVA: analysis of covariance; AST: aspartate aminotransferase; BDI: Beck Depression Inventory; BMI: body mass index; BP: blood pressure; CDC: Centers for Disease Control and Prevention; CFS: chronic fatigue syndrome; CRIS: Clinical Research Information Service; CXR: chest radiography; ECG: electrocardiography; ECT: explanatory clinical trial; EQ-5D: EuroQol-5 Dimension; FAS: full analysis set; FSS: Fatigue Severity Scale; FT4: free thyroxine; Hb: hemoglobin; hCG: human chorionic

gonadotropin; Hct: hematocrit; ICF: idiopathic chronic fatigue; ITT: intention to treat; LOCF: last observation carried forward; MFS: Multiple Fatigue Scale; MMRM: mixed model for repeated measures; NRS: Numeric Rating Scale; PCT: pragmatic clinical trial; PPS: per-protocol set; SAS: Strategic Applications Software; SRI: Stress Response Inventory; TCM: traditional Chinese medicine; TFT: fhyroid function test; TSH: thyroid stimulating hormone; VAS: Visual Analogue Scale; WBC: white blood cells.

\section{Competing interests}

The authors declare that they have no competing interests.

\section{Authors' contributions}

JEK contributed to the conception and design of the study and wrote the manuscript. BKS, JBC, HJK, THK, JHK, KMS, SL, YHB, and KEH participated in the design of the study and helped to draft the manuscript. MHL and KWK conducted statistical design of the study and wrote part of the statistical methods. SYJ, ARK, MSS, HJJ, HJP, and SPK provided technical advice and contributed to a critical review of the manuscript. SMC participated in the design of the study as the principal investigator and provided a critical review of the manuscript. All authors read and approved the final manuscript.

\section{Acknowledgments}

This study was supported by the Korea Institute of Oriental Medicine (K12010 and K15122). The funders had no role in design, in the collection, analysis, and interpretation of data; in the writing of the manuscript; or in the decision to submit the manuscript for publication. The authors would like to acknowledge the following people for their help in the study: Gwang-Chen Park and Jae-Hong Kim at Gwangju Oriental Hospital and Yu-Rim Park at Jecheon Oriental Hospital gave suggestions for addressing problems encountered during the trial period, and Min-Jung Kim and Pil-Kun Kim at Kyung Hee University Hospital at Gangdong, and Yeon-Hee Ryu at Korea Institute of Oriental Medicine, provided helpful advice on the research design.

\section{Author details}

${ }^{1}$ Acupuncture, Moxibustion \& Meridian Research Group, Korea Institute of Oriental Medicine, Daejeon, South Korea. ${ }^{2}$ Department of Acupuncture \& Moxibustion, Kyung Hee University Hospital at Gangdong, Seoul, South Korea. ${ }^{3}$ Department of Oriental Rehabilitation Medicine, Gwangju Oriental Hospital of Dongshin University, Gwangju, South Korea. ${ }^{4}$ Department of Oriental Gynecology, Jecheon Oriental Hospital of Semyung University, Jecheon, South Korea. ${ }^{5}$ College of Korean Medicine, Gachon University, Seongnam, South Korea. ${ }^{6}$ Department of Acupuncture \& Moxibustion, Kyung Hee University Medical Center, Seoul, South Korea. ${ }^{7}$ Department of Acupuncture \& Moxibustion, Daejeon Oriental Hospital of Daejeon University, Daejeon, South Korea. ${ }^{8}$ Department of Korean Medicine, Nurije Korean Medical Clinic, Daejeon, South Korea.

Received: 1 February 2015 Accepted: 9 July 2015 Published online: 26 July 2015

\footnotetext{
References

1. Jorgensen R. Chronic fatigue: an evolutionary concept analysis. J Adv Nurs. 2008;63:199-207
} 
2. Fukuda K, Straus SE, Hickie I, Sharpe MC, Dobbins JG, Komaroff A. The chronic fatigue syndrome: a comprehensive approach to its definition and study. International Chronic Fatigue Syndrome Study Group. Ann Intern Med. 1994;121:953-9.

3. Adams D, Wu T, Yang X, Tai S, Vohra S. Traditional Chinese medicinal herbs for the treatment of idiopathic chronic fatigue and chronic fatigue syndrome. Cochrane Database Syst Rev. 2009:4:cd006348.

4. Son CG. Review of the prevalence of chronic fatigue worldwide. J Korean Oriental Med. 2012;33:25-33.

5. Afari N, Buchwald D. Chronic fatigue syndrome: a review. Am J Psychiatry. 2003:160:221-36.

6. Choi DH, Kim CH, Shin HC, Park YW, Sung EJ, Lee KH. Patterns of complementary and alternative medicine therapies in patients with chronic fatigue or pain. Korean J Farm Med. 2009;30:182-9 [article in Korean].

7. Wang YY, Li XX, Liu JP, Luo H, Ma LX, Alraek T. Traditional Chinese medicine for chronic fatigue syndrome: a systematic review of randomized clinical trials. Complement Ther Med. 2014;22:826-33.

8. $\quad$ Zhang W, Peng WN, Liu ZS. Acupuncture for chronic fatigue syndrome: systematic review. J Evidence-Based Med. 2009;9:41-7 [article in Chinese].

9. Wang JJ, Song YJ, Wu ZC, Chu XO, Wang XH, Wang XJ, et al. A meta analysis on randomized controlled trials of acupuncture treatment of chronic fatigue syndrome. Zhen Ci Yan Jiu. 2009:34:421-8 [article in Chinese]

10. Cheng ZD, Chen YG, Zhang T, Che XJ. Systematic review on acupuncture and moxibustion treatment of chronic fatigue syndrome. Chin J Inf Tradit Chin Med. 2011;18:30-2 [article in Chinese].

11. Wang T, Zhang Q, Xue X, Yeung A. A systematic review of acupuncture and moxibustion treatment for chronic fatigue syndrome in China. Am J Chin Med. 2008:36:1-24.

12. Kim JE, Kang KW, Kim AR, Kim JH, Kim TH, Park HJ, et al. Acupuncture for chronic fatigue syndrome and idiopathic chronic fatigue: a pilot randomized controlled trial. J Korean Acu Mox Soc. 2012;29:109-18 [article in Korean].

13. Kim SH, Park HJ, Park HA, Jang JH, Hwang KS, Lee SY. The clinical study on the effect of Sa-am acupuncture treatment for patients with fatigue. J Korean Acu Mox Soc. 2007;24:149-57 [article in Korean].

14. World Health Organization. WHO International Standard Terminologies on Traditional Medicine in the Western Pacific Region. 1st ed. Manila: World Health Organization; 2007.

15. Kim JE, Hong KE, Kim HJ, Choi JB, Baek YH, Seo BK, et al. An open-label study of effects of acupuncture on chronic fatigue syndrome and idiopathic chronic fatigue: study protocol for a randomized controlled trial. Trials. 2013;14:147.

16. General Assembly of the World Medical Association. World Medical Association Declaration of Helsinki. Ethical principles for medical research involving human subjects. J Am Coll Dent. 2014;8:14-8.

17. Yi JH, Lee JH, Park SM, Kim YC, Lee JH, Woo HJ. Clinical investigation and follow-up monitoring of fatigue patients visiting the oriental medical hospital. J Korean Oriental Med. 2000;21:55-63 [article in Korean].

18. Shim SY, Park HJ, Lee JM, Lee HS. An overview of pain measurements. Korean J Acu. 2007;24:77-97 [article in Korean].

19. Korean Acupuncture and Moxibustion Society. Acupuncture and Moxibustion. 2nd ed. Paju: Jipmoondang; 2008

20. Flaws B. The Classic of Difficulties. 6th ed. Boulder: Blue Poppy Press; 2006

21. Macpherson H. Pragmatic clinical trials. Complement Ther Med. 2004;12:136-40.

22. Yun YJ, Kang KW, Choi SM. An analysis of clinical trials for understanding and application of pragmatic clinical trial. J Korean Acu Mox Soc. 2009;26:105-16 [article in Korean]

23. Krupp LB, LaRocca NG, Muir-Nash J, Steinberg AD. The fatigue severity scale. Application to patients with multiple sclerosis and systemic lupus erythematosus. Arch Neurol. 1989;46:1121-3.

24. Chung Kl, Song $\mathrm{CH}$. Clinical usefulness of Fatigue Severity Scale for patients with fatigue, and anxiety or depression. Korean J Psychiatr Med. 2001;9:164-73 [article in Korean].

25. Choi SM, Kang TY, Woo JM. Development and validation of a modified form of the Stress Response Inventory for workers. J Korean Neuropsychiatr Assoc. 2006:45:541-3 [article in Korean].

26. Rhee MK, Lee YH, Park SH, Sohn CH, Chung YC, Hong SK, et al. A standardization study of Beck Depression Inventory I - Korean version
(K-BDI): reliability and factor analysis. Korean. J Psychopathol. 1995;4:77-95 [article in Korean].

27. Devilly GJ, Borkovec TD. Psychometric properties of the credibility/ expectancy questionnaire. J Behav Ther Exp Psychiatry. 2000;31:73-86.

28. Lewis JA. Statistical principles for clinical trials (ICH E9): an introductory note on an international guideline. Stat Med. 1999;18:1903-42.

29. Mallinckrodt CH, Lane PW, Schnell D, Peng Y, Mancuso JP. Recommendations for the primary analysis of continuous endpoints in longitudinal clinical trials. Drug Inf J. 2008;42:303-19.

30. Chang SJ. Fatigue. In: The Korean society of preventive medicine. Standardization and measurement of health. Seoul: Gyechuk Press; 2000. p. 141-81 [article in Korean].

31. Gregori D, Baldi I, Frigo AC, Guardabasso V. Why, when and how to adjust for multiplicity in clinical trials: a perspective on regulatory activities. Biomedical Statistics Clin Epidemiol. 2010;4:27-34.

32. Dunnett CW. A multiple comparison procedure for comparing several treatments with a control. J Am Stat Assoc. 1955:50:1096-121.

33. Dittner AJ, Wessely SC, Brown RG. The assessment of fatigue: a practical guide for clinicians and researchers. J Psychosom Res. 2004;56:157-70.

34. Whitehead $L$. The measurement of fatigue in chronic illness: a systematic review of unidimensional and multidimensional fatigue measures. J Pain Symptom Manage. 2009:37:107-28.

35. Group E. EuroQol-a new facility for the measurement of health-related quality of life. Health Policy. 1990;16:199-208.

36. Cairns R, Hotopf M. A systematic review describing the prognosis of chronic fatigue syndrome. Occup Med (Lond). 2005;55:20-31.

37. Tanaka M, Watanabe $Y$. A new hypothesis of chronic fatigue syndrome: co-conditioning theory. Med Hypotheses. 2010;75:244-9.

38. Friedberg F, Jason LA. Chronic fatigue syndrome and fibromyalgia: clinical assessment and treatment. J Clin Psychol. 2001;57:433-55.

39. Lane P. Handling drop-out in longitudinal clinical trials: a comparison of the LOCF and MMRM approaches. Pharm Stat. 2008;7:93-106.

40. Siddiqui O, Hung HM, O'Neill R. MMRM vs. LOCF: a comprehensive comparison based on simulation study and 25 NDA datasets. J Biopharm Stat. 2009;19:227-46.

41. Rothwell PM. Treating individuals 2. Subgroup analysis in randomised controlled trials: importance, indications, and interpretation. Lancet. 2005:365:176-86.

42. Son CG. Analysis of patients visiting an oriental hospital with idiopathic chronic fatigue or chronic fatigue syndrome. J Korean Med. 2013;34:119-25 [article in Korean].

43. Kwak KK, Cho JH, Son CG. Study on chronic fatigue syndrome from oriental medicine point of view. Korean J Orient Int Med. 2008;29:962-9 [article in Korean].

44. Lee BH, Lee SN, Kim DJ, Kim JW, Lim SC, Jung TY, et al. A study on the fixed- and transformed-pattern of Sa-am acupuncture treatment. J Korean Acu Mox Soc. 2008;25:1-9 [article in Korean]

45. Kim DH. Saamdoin Acupuncture Therapy. 1st ed. Busan: Sogang Press; 2001

46. Cho MS, Ahn CB. A review of the manipulation methods and the supplementation and draining method in acupuncture. J Korean Acu Mox Soc. 1996;13:404-21 [article in Korean].

47. Yan CL, Zhu YL. The Treatment of External Diseases with Acupuncture and Moxibustion. 2nd ed. Boulder: Blue Poppy Press; 2007.

48. An JR, Lee IS. The study of Saamchimbeop's method of reinforcement and reduction. J Oriental Rehab Med. 2009;19:113-23 [article in Korean].

49. Ernst $G$, Strzyz $H$, Hagmeister $H$. Incidence of adverse effects during acupuncture therapy-a multicentre survey. Complement Ther Med. 2003;11:93-7.

50. Macpherson H, Scullion A, Thomas KJ, Walters S. Patient reports of adverse events associated with acupuncture treatment: a prospective national survey. Qual Saf Health Care. 2004;13:349-55.

51. White A. The safety of acupuncture-evidence from the UK. Acupunct Med 2006;24:53-7.

52. Di Blasi Z, Harkness E, Ernst E, Georgiou A, Kleijnen J. Influence of context effects on health outcomes: a systematic review. Lancet. 2001;357:757-62.

53. Larun L, Malterud K. Identity and coping experiences in Chronic Fatigue Syndrome: A synthesis of qualitative studies. Patient Educ Couns. 2007;69:20-8.

54. Hewlett S, Dures E, Almeida C. Measures of fatigue: Bristol Rheumatoid Arthritis Fatigue Multi-Dimensional Questionnaire (BRAF MDQ), Bristol Rheumatoid 
Arthritis Fatigue Numerical Rating Scales (BRAF NRS) for severity, effect, and coping, Chalder Fatigue Questionnaire (CFQ), Checklist Individual Strength (CIS20R and CIS8R), Fatigue Severity Scale (FSS), Functional Assessment Chronic Illness Therapy (Fatigue) (FACIT-F), Multi-Dimensional Assessment of Fatigue (MAF), Multi-Dimensional Fatigue Inventory (MFI), Pediatric Quality Of Life (PedsQL) Multi-Dimensional Fatigue Scale, Profile of Fatigue (ProF), Short Form 36 Vitality Subscale (SF-36 VT), and Visual Analog Scales (VAS). Arthritis Care Res. 2011;63 Suppl 11:S263-86.

55. Tiesinga $L$, Dassen TW, Halfens RJ. Fatigue: a summary of the definitions, dimensions, and indicators. Nurs Diagn. 1996;7:51-62

\section{Submit your next manuscript to BioMed Central} and take full advantage of:

- Convenient online submission

- Thorough peer review

- No space constraints or color figure charges

- Immediate publication on acceptance

- Inclusion in PubMed, CAS, Scopus and Google Scholar

- Research which is freely available for redistribution 